\title{
Estado del Arte de la Evaluación de Tecnologías en Salud en América Latina
}

\author{
State of the Art of the Evaluation of Health Technologies in Latin America
}

\author{
F. M. Martínez-Licona, M. R. Ortiz-Posadas, M. R. Ortiz-Pedroza
}

Universidad Autónoma Metropolitana Iztapalapa

\section{RESUMEN}

El surgimiento y desarrollo de las tecnologías en salud ha hecho que se intensifique el papel de su evaluación en los últimos años, provocando un interés creciente en la Evaluación de Tecnologías en Salud (ETES) en América Latina, a través de intentos por proporcionar información útil al tomador de decisiones. Este artículo presenta una revisión del estado del arte de ETES en Latinoamérica, a partir del análisis de publicaciones en revistas y eventos especializados. Se abordaron tres aspectos: elementos de evaluación, métodos que se aplican y políticas resultantes. Se encontraron los siguientes aspectos de evaluación: seguridad, eficacia, efectividad y eficiencia, económico-financieros, clínicos y técnicos, estos últimos con mayor desarrollo. Con respecto a los métodos, se encontraron propuestas de estrategias empíricas para el análisis de la información y la toma de decisiones. La generación de políticas públicas relacionadas con la ETES en América Latina es incipiente, apenas se están identificando las problemáticas nacionales y las estrategias a seguir para su solución. Por lo tanto, se aprecia una necesidad de seguir trabajando en el desarrollo de políticas, estrategias y métodos de ETES en la región Latinoamericana que permitan responder a las problemáticas en salud de la población de cada país.

PAlabras Clave: Evaluación de tecnologías de salud; Ingeniería Clínica; Elementos de Evaluación; Métodos de evaluación; Políticas Públicas 


\section{ABSTRACT}

The emergence and development of health technologies have intensified the role of their evaluation in recent years, causing a growing interest in the Evaluation of Health Technologies (ETES) in Latin America, through attempts to provide useful information to the decision-maker. This article presents a review of the state of the art of ETES in Latin America, from the analysis of publications in journals and specific events. Three aspects were addressed: evaluation elements, methods that are applied, and resulting policies. The following evaluation aspects were found: safety, efficacy, effectiveness and efficiency, economic-financial, clinical and technical, the latter with more significant development. Concerning the methods, proposals for empirical strategies for the analysis of information and decision making were found. The generation of public policies related to the HTA in Latin America is under development, national problems are hardly being identified, and the strategies to be followed for their solution. Therefore, there is a need to continue working on the development of policies, and methods of HTA in the Latin American region that allow responding to the health problems of the population of each country.

KEYwORDS: Health Technologies Assessment; Clinical Engineering; Elements of Assessment; Assessment Methods; Public Policies

\section{Correspondencia}

DESTINATARIO: Fabiola Margarita Martínez Licona

INSTITUCIÓN: Universidad Autónoma Metropolitana

DIRECCIÓN: Av. Avenida San Rafael Atlixco \#186,

Col. Vicentina, C. P. 09340, Del. Iztapalapa,

Ciudad de México, México

CORREO ELECTRÓNICO: fmml@xanum.uam.mx

\section{Fecha de recepción:}

15 de junio de 2019

Fecha de aceptación:

15 de agosto de 2019 


\section{INTRODUCCIÓN}

La velocidad con la que se desarrollan y producen las tecnologías en materia de salud requiere de planeación y estrategias que, con la ayuda de métodos y herramientas, puedan contender con los aspectos que se involucran en su evaluación. El papel de la Evaluación de Tecnologías en Salud (ETES) se ha intensificado en los últimos años. La Organización Mundial de la Salud (OMS) le otorgó un carácter estratégico y global a partir de las resoluciones que en este tema ha dado a conocer desde hace más de una década. Este organismo define a la ETES como la evaluación sistemática de las propiedades, efectos u otros impactos de la tecnología de la salud ${ }^{[1]}$. Entre sus finalidades se encuentran el apoyo y la promoción del desarrollo, difusión, comprensión y utilización como una base científica para la toma de decisiones. Como consecuencia, el interés en la ETES ha aumentado en Latinoamérica reflejándose en numerosos intentos por proporcionar información de utilidad al tomador de decisiones. De igual manera debe hacerse notar las acciones en torno a la organización de las acciones y productos resultantes de estos procesos.

Con el fin de comprender la complejidad que implica evaluar el impacto de una tecnología en el ámbito de la salud, se tiene que partir de las bases conceptuales. Evaluar signifca asignar un valor a algo. Si lo que se desea es la asignación del valor que tiene una tecnología en este contexto, entonces se deberán incorporar todos los aspectos que estén relacionados, incluyendo el técnico, clínco, económico, social, ético, jurídico, comercial y regulatorio, entre otros. La sistematización de esta función requiere de aproximaciones bien estructuradas que permitan la asignación correcta de dicho valor, empezando con la definición del valor a encontrar en cada ámbito de consideración, la forma en que éste se determinará y su propósito.

En este artículo se presenta una revisión del estado del arte en el tema de la ETES en América Latina. Para tal fin se analizaron las publicaciones en revistas y eventos especializados con la intención de analizar tres aspectos: los elementos de evaluación, los métodos que se aplican en la evaluación y las políticas resultantes de la misma.

\section{¿QUÉ SE EVALÚA? ELEMENTOS DE LA EVALUACIÓN DE TECNOLOGÍAS EN SALUD}

Aun cuando históricamente se han dado definiciones de tecnologías en salud o tecnologías sanitarias, así como definiciones y propósitos para la ETES, como las establecidas por la OMS o por la Red Internacional de Agencias de Evaluación de Tecnología en Salud [2], la interpretación que cada país le ha dado a dichas definiciones y a los propósitos de la evaluación ha ocasionado que actualmente existan diferentes matices y niveles de acción de las ETES, que dan lugar al cuestionamiento sobre los propósitos generales y específicos que se han considerado en los diferentes grupos involucrados al realizar un proceso de este tipo.

Con respecto a los niveles de acción de una evaluación se pueden identificar los siguientes:

N1. Evaluación regulatoria realizada por las agencias de control de medicamentos o de equipos médicos nacionales con el fin de generar un registro nacional.

N2. ETES para políticas en salud realizada por instancias de evaluación de tecnología nacionales o regionales para su utilización o inclusión en planes de atención o para proporcionar algún beneficio en la misma.

N3. ETES en hospitales públicos y privados con el propósito de incorporar tecnologías acordes al perfil institucional.

\section{N4. Evaluación para la adquisición de equipos reali-} zada por departamentos de logística o de gestión tecnológica para la selección más adecuada dentro de un grupo de ofertas disponibles (evaluación pre-compra). 


\section{N5. Evaluación durante la fase de utilización dentro} del ciclo de vida de la tecnología, la cual idealmente debería de ser realizada o fuertemente soportada por el departamento de ingeniería biomédica (DIB) del hospital, con el propósito de analizar, principalmente, el grado de funcionalidad y seguridad que la tecnología presenta después de determinado tiempo de uso en comparación con las especificaciones del fabricante.

\section{N6. La evaluación de tecnología para la disposición}

final o el reemplazo de la tecnología, generalmente también soportada por el DIB de las instituciones de atención a la salud con el propósito de determinar de manera oportuna, objetiva y eficiente la disposición final de la misma.

Por otra parte, en reportes sobre procesos de ETES se puede encontrar la mención de diferentes aspectos a analizar para la evaluación, los cuales dependerán del nivel a abordar. En el caso de la evaluación regulatoria (N1) suelen considerarse aspectos de seguridad y de eficacia; en las ETES para políticas en salud (N2) se llegan a considerar la seguridad, la efectividad, el análisis costo-efectividad y los impactos sociales; para ETES en hospitales (N3) suelen analizarse aspectos de seguridad, efectividad, costo-efectividad, aspectos éticos e impacto organizacional; mientras que en procesos de evaluación para adquisición de equipos (N4) se han considerado factores clínicos, factores técnicos $\mathrm{y}$ factores financieros ${ }^{[3]}$.

En esta sección se presentan ejemplos de aplicación de los aspectos generales para realizar las ETES en Latinoamérica, así como la mención o descripción de los elementos específicos utilizados para su análisis.

\section{Seguridad}

La seguridad del paciente es uno de los ejes de la evaluación de toda tecnología en salud por lo que suele considerarse en todos los niveles de acción. Además, la seguridad consistentemente estará relacionada directa o indirectamente con más de un aspecto analizado durante una ETES como lo es el clínico o el técnico. En este sentido, son varios los autores que en sus reportes sobre visiones generales o propuestas específicas de procesos de ETES mencionan su importancia como

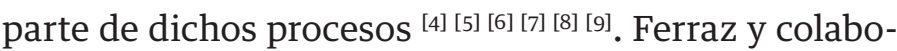
radores, por ejemplo, reportan que en la opinión de un grupo de personas involucradas en la economía de salud, la seguridad es uno de los tres criterios más relevantes en un proceso de ETES ${ }^{[10]}$ mientras que en Ruiz y colaboradores se refuerza esta opinión a través de líderes de la gestión de la tecnología y de la gestión de la seguridad del paciente en Colombia ${ }^{[11]}$.

Fors y Peña describen el proceso desde la fase de comercialización hasta el registro sanitario de la tecnología (relacionados con los niveles de acción "macro" N1 y N2) y mencionan como necesaria la evaluación de la seguridad desde la realización de los estudios preclínicos ${ }^{[12]}$. Por otra parte, en el trabajo de Ardila y colaboradores se presenta una propuesta de método de incorporación estratégica de tecnología biomédica para instituciones de salud (niveles N3 y N4) y se menciona que la seguridad es uno de los criterios de mayor importancia para los ingenieros clínicos de instituciones prestadoras de servicios de Colombia, donde el análisis de riesgos y el historial de recalls asociados al equipo, son factores importantes a tomar en cuenta antes de tomar una decisión de compra. Asimismo se menciona que la evaluación de la seguridad puede tener dos enfoques, el primero toma en cuenta el riesgo inminente de un dispositivo según su complejidad y forma de uso y el segundo cuantifica la probabilidad que nace de experiencias pasadas y se recolecta por medio de la búsqueda de información en bases de datos, agencias o instituciones como la FDA, ECRI y el INVIMA entre otros organismos de evaluación y certificación ${ }^{[13]}$.

En otros estudios de ETES para procesos de adquisición (N4), se ha ubicado a la seguridad como parte de la evaluación técnica, relacionándola con el cumpli- 
miento de las especificaciones técnicas del fabricante [14, 15], o bien como parte de la evaluación clínica si se orienta hacia la seguridad en la operación y por consiguiente en el paciente ${ }^{[14]}$. De manera similar, pero con un enfoque de renovación tecnológica Duque y coautores presentan una propuesta de protocolo de evaluación de equipos médicos en el que se considera relevante la evaluación de los sistemas de seguridad dentro del rubro de la evaluación técnico-funcional ${ }^{[16]}$.

Uno de los aspectos de seguridad que no debe faltar en un proceso de ETES, cuando se trata de equipos electromédicos es la seguridad eléctrica, en la que se prueba, por ejemplo, que los equipos médicos no sobrepasen los límites de corrientes de fuga aceptados como seguros por alguna instancia regulatoria tal como la Comisión Electrotécnica Internacional (IEC por sus siglas en inglés) en sus apartados IEC60601 para pruebas de ensayo (pre-comercialización) y IEC62353 para pruebas de rutina (en la fase de utilización).

En este sentido, Correa y colaboradores, presentan un protocolo para la evaluación del desempeño de equipos en la fase de utilización (Nivel N5) como una herramienta de vigilancia importante que contribuye en sí mismo a la seguridad del paciente y de manera más contundente al incluir el análisis de la seguridad eléctrica como una de las pruebas generales a realizar ${ }^{[17]}$.

Otro ejemplo relacionado con la seguridad eléctrica es el que proponen Acevedo y colaboradores, como parte de los elementos de evaluación considerados en su propuesta de diseño de protocolo para la validación de tecnología médica, el cual fue aplicado a los módulos de un monitor de signos vitales, probando que la batería funcione correctamente después de desconectar el equipo de la corriente eléctrica ${ }^{[18]}$.

Otro elemento relevante para la evaluación de la seguridad es el análisis de la ocurrencia de eventos adversos vinculados con la tecnología médica, relacio- nado a su vez con la tecnovigilancia y la gestión de riesgos ${ }^{[11]}[19]$. En el estudio de Arregui y colaboradores, se analizan 112 auditorías de instalaciones eléctricas y seguridad de equipos médicos clasificando el nivel de riesgo, verificando el cumplimiento de normas y analizando el estado de mantenimiento y la documentación sobre procedimientos de incorporación de equipos, la documentación de salidas e intervenciones preventivas y correctivas, la de pruebas de calibración y la disponibilidad de manuales, entre otros [20]. Sánchez y colaboradores a su vez, presentan los resultados de un estudio sobre las prácticas de tecnovigilancia y gestión de riesgos clínicos en la región suroccidental de Colombia, en el entendido que las instituciones deberían de adoptar e implementar la tecnovigilancia no solo con el objetivo de cumplir con los requisitos mínimos exigidos por la ley sino como una oportunidad de mejoramiento constante y como un método para reforzar la seguridad de los pacientes ${ }^{[19]}$.

\section{Eficacia}

En el contexto de la ETES se entiende por eficacia a la capacidad de la tecnología para producir efectos/resultados benéficos/esperados en condiciones ideales, lo cual implica la realización de pruebas de desempeño, generalmente llevadas a cabo en laboratorios especializados y bajo condiciones controladas. Por lo anterior, es más probable encontrar que la eficacia sea evaluada dentro de procesos de ETES previos a la etapa de comercialización o en la fase de incorporación de la tecnología (N1 a N3).

El concepto de eficacia es muy abierto en el sentido de que se pueden analizar una gran variedad de efectos o resultados, así como gran variedad de características para calificarlos como benéficos o precisos. En algunos reportes de ETES únicamente se hace mención de la inclusión de este aspecto en el análisis, sin que se especifique con claridad qué elementos se utilizaron para hacerlo mientras que hay otros que los describen con cierto nivel de detalle. La diversidad de elementos 
de evaluación encontradas en los reportes de ETES depende claramente del tipo de tecnología analizada, pero también de las motivaciones particulares de los sujetos involucrados en la evaluación y probablemente de la interpretación que se le dio a la definición del aspecto evaluado (p. ej. el de eficacia).

Son varios los estudios de revisión o de análisis de ETES así como trabajos con propuestas metodológicas que incorporan la variable eficacia en el análisis. En uno de ellos, se considera a la eficacia como uno de los tres aspectos más relevantes junto con la seguridad y la relevancia de la enfermedad; también se ha incluido como uno de los elementos presentes en los protocolos propuestos para la evaluación de equipos médicos; se ha mencionado como parte de los estudios pre-clínicos necesarios durante el desarrollo de equipos y de productos farmacéuticos y biotecnológicos, entre otros [3] [4] [6] [7] [9] [10] [12] [14] [16] [21] [22].

\section{Efectividad}

La efectividad, similar a la eficacia, es la capacidad de la tecnología para producir efectos/resultados benéficos/esperados, pero en este caso en condiciones reales, por ejemplo cuando la tecnología es utilizada u operada por el usuario y en el entorno destinado para su uso cotidiano como en el área clínica en la que se utiliza un cierto equipo médico sometido a evaluación.

La evaluación de la efectividad, por tanto, se considera más frecuentemente en procesos de ETES de los niveles N2 a N6. En un análisis del contenido de los programas de ETES en Latinoamérica, Arellano y cols. identificaron que la efectividad clínica junto con la evaluación económica son de los aspectos más importantes a considerarse, ya que son los que en mayor medida se presentan en la documentación analizada (66\% de los documentos) ${ }^{[23]}$. Lo anterior se constata al encontrar que se incluye la evaluación de la efectividad en trabajos sobre ETES para los diferentes niveles de evaluación [3] [4] [6] [7] [9] [10] [14] [16] [21] [22] [24] [25].

\section{Eficiencia}

La eficiencia es una medida de la relación entre resultados obtenidos o consecuencias derivadas del uso de una tecnología en salud y los recursos invertidos en ello. Se mide en función del grado de utilización real y efectiva de los recursos en relación con la utilización programada (utilización efectiva/utilización programada del recurso) ${ }^{[4]}$. Un ejemplo del análisis de la eficiencia como parte de los procesos de ETES se presenta en el caso del diseño y desarrollo de un protocolo para la validación de la calidad en el diseño de tecnología médica. En este estudio se considera a la eficiencia como uno de los elementos de evaluación enfocado a probar que una empresa que diseña y fabrica dispositivos logra que sus productos se encuentren libres de defectos y cumplen conscientemente las especificaciones planteadas por la empresa y las normas. En este ejemplo, el protocolo propuesto en la forma de lista de verificación (checklist) fue aplicado para la evaluación del desempeño de los módulos de un monitor de signos vitales y la eficiencia en la usabilidad fue medida en términos de los recursos empleados (esfuerzo y tiempo) para la operación de los módulos del monitor ${ }^{[18]}$.

\section{Aspectos económico-financieros}

Uno de los aspectos que en mayor medida se toma en cuenta en el contexto de la ETS es la evaluación de los aspectos económicos y financieros. La evaluación económica es un conjunto de técnicas que se utilizan para comparar las opciones abiertas para los tomadores de decisiones en una situación de elección entre un conjunto de posibles cursos de acción. Las técnicas para evaluaciones económicas tienen en común que los recursos utilizados son comparados con los resultados, los que se pueden expresar como eficacia, efectividad, utilidad y beneficio ${ }^{[4]}$. Una evaluación económica puede ser parcial o total. En una evaluación parcial se realiza un análisis de costos y en una total se puede buscar la minimización de los costos, o bien hacer análisis costo-efectividad, costo-utilidad o costo-beneficio ${ }^{[23]}$. 
Lo que se espera de una evaluación económica es que aporte una adecuada evidencia del valor terapéutico y social de las nuevas tecnologías en comparación con su costo ${ }^{[26]}$. A manera de ejemplo se puede mencionar que en una lista de verificación para describir las consecuencias económicas de la introducción de una nueva tecnología específica para un grupo de pacientes y servicio clínico específico, se pueden incluir preguntas orientadas a conocer cuáles serán los aportes de esa tecnología en los próximos años, cuál será el costo adicional por paciente al año con el uso de la nueva tecnología, el costo total aumentado del hospital en los próximos años, la reducción y contención de costos, y los costos por trámites ${ }^{[27]}$.

La evaluación costo-efectividad es útil para comparar dos o más alternativas, donde los recursos se expresan en unidades monetarias y los efectos sobre la salud en unidades específicas no monetarias de efectividad (medidas de efectividad). En este tipo de análisis se pueden expresar los efectos en las mismas unidades que las utilizadas en los ensayos clínicos o en la práctica clínica, pero sólo se puede seleccionar entre opciones similares y que tengan efectos medidos en las mismas unidades. Los resultados de un análisis costo-efectividad pueden presentarse de dos formas: Costo-efectividad media obtenido a partir de la relación costo/efectividad y con unidades de pesos por unidad de efectividad o bien, Costo-efectividad incremental, obtenido mediante la relación (Costo A - Costo B)/(Efecto A - Efecto B) ${ }^{[28]}$.

Este tipo de análisis también suele realizarse como parte de las ETES para los diferentes niveles de evaluación [4] [7] [9] [14] [21] [25] [26] [27] [29] [30] [31] [32]. Sin embargo, en los reportes de evaluación en los que se incluye este aspecto suele indicarse, en términos generales, que el análisis costo-efectividad debe ser contemplado en la ETES, o que se realizó dicho tipo de análisis sin especificar con claridad los elementos tomados en cuenta para obtener medidas analíticas cuantitativas.

\section{Aspectos clínicos}

La evaluación clínica se refiere al proceso en el que se recurre a miembros del personal clínico para evaluar la tecnología médica en el entorno en el que es utilizada, con el propósito de obtener la información referente al cumplimiento de sus características. Este elemento de evaluación, entendido como la evaluación del impacto o beneficio clínico, es el reconocido como uno de los más importantes en una ETES y para todos los niveles de evaluación ${ }^{[29]}$. El beneficio clínico puede darse en el diagnóstico, la terapia, el reemplazo de un órgano o en el monitoreo de la evolución del paciente ${ }^{[33]}$. El perfil clínico de un producto se evalúa de manera explícita en función de su beneficio relativo incremental con respecto a la atención estándar actual ${ }^{[29]}$. No obstante lo anterior, el estudio de Lingg y cols. sobre los puntos de vista de los usuarios finales respecto al proceso de compra de dispositivos médicos (Nivel N4), muestra que el sentir de los usuarios finales es que los tomadores de decisiones suelen enfocarse en las especificaciones mecánicas y técnicas y no se integra un espectro de criterios de decisión tal como el impacto clínico a corto y largo plazo, ni se integra adecuadamente a los especialistas en la toma de decisiones ${ }^{[32]}$. La influencia de factores del entorno en el que se usa un dispositivo médico que repercutan en la medición también debe de ser un elemento a considerar en una ETES. Acevedo y cols., por ejemplo, en su propuesta de protocolo de validación de tecnología médica aplicado a los módulos de un monitor incluyen el análisis de la incidencia de fallas en la medición del módulo de oximetría asociados a la interferencia de la luz solar, de la luz artificial y a la presencia de hematomas ${ }^{[18]}$. Algunos de los elementos que se propone considerar en una evaluación clínica en procesos de adquisición de la tecnología (nivel de evaluación N4) son los siguientes: el cumplimiento de las características, calidad tecnológica, relevancia clínica, experiencia y satisfacción total. Mientras que algunos criterios de evaluación clínica se enumeran a continuación: el cumplimiento de la necesidad, la visibilidad de controles, la facilidad para com- 
prender y escuchar señales de alarma, la adaptabilidad de la tecnología en el proceso clínico, la comodidad para realizar su limpieza para usos futuros, la calidad de la capacitación tecnológica, la facilidad de conexión, el reconocimiento y manejo de los accesorios de la tecnología y la interoperabilidad ${ }^{[14]}$.

Se puede notar que algunos de los criterios listados anteriormente están relacionados con aspectos de usabilidad, entendiéndola como la capacidad de un software o dispositivo para ser comprendido, aprendido, usado y ser atractivo para el usuario, en condiciones específicas de uso ${ }^{[34]}$. En este sentido se pueden agregar los siguientes criterios propuestos por Acevedo y cols.: la facilidad del aprendizaje para operarlo, la ausencia de errores del sistema y la facilidad del sistema para ser recordado ${ }^{[18]}$ y los contemplados en el estudio de Cabrera y cols. como son, los requisitos de conocimiento del usuario, el estado físico de la pantalla y la respuesta del dispositivo a las acciones del operador ${ }^{[34]}$.

\section{Aspectos técnicos}

La evaluación técnica se define como el proceso de revisión de las especificaciones técnicas del sistema o equipo biomédico, entre las que destaca su funcionamiento, funcionalidad o desempeño, seguridad, requerimientos eléctricos y compatibilidad.

Con este tipo de evaluación se busca asegurar que las tecnologías en salud cumplan con las especificaciones del fabricante y con los requisitos de seguridad del hospital y de los organismos reguladores. Como parte de este tipo de evaluaciones existen pruebas de laboratorio para valorar la funcionalidad, aspectos físicos para aprovechar los recursos de forma segura para el paciente y el operador del equipo ${ }^{[14]}$.

A manera de ejemplo se puede mencionar la herramienta de ETES propuesta por Chavarría y cols., la cual fue implementada para un monitor de signos vitales y que incorpora elementos de evaluación técnica a manera de listado de especificaciones que el usuario debe de calificar numéricamente entre uno y cinco de acuerdo a su nivel de importancia, donde 5 corresponde a una calificación muy alta y 1 es deficiente para el equipo en dicha especificación ${ }^{[15]}$. De manera similar, en el estudio relativo al diseño de un protocolo para la validación de la calidad en el diseño de tecnología médica aplicado a monitores de signos vitales, uno de los elementos de evaluación fue el desempeño y funcionalidad, verificando que el equipo mida correctamente en el intervalo de valores descrito en el manual técnico, es decir, la calibración del sistema, así como la evaluación del funcionamiento adecuado de las alarmas y la medición de porcentaje de error de las mediciones ${ }^{[18]}$. Duque y cols., por otra parte, en su propuesta de protocolo de evaluación de equipos médicos considera aspectos técnicos como la edad, la apariencia, los componentes, los sistemas de seguridad, el entorno, el nivel de riesgo, el número de años con soporte de refacciones, de consumibles y técnico humano, manuales, frecuencia de mantenimiento, vida útil estimada, número de fallas y complejidad del mantenimiento ${ }^{[16]}$. Ramírez y cols. proponen un modelo de gestión y evaluación de conformidad que caracteriza las exigencias establecidas en las normas para integrar los requerimientos del control metrológico legal aplicado a equipos médicos (aseguramiento de las mediciones) y contiene el proceso de soporte técnico que califica a los equipos según si aplica o no el soporte metrológico legal. En esta propuesta se consideran elementos de evaluación tal como el cumplimiento con las especificaciones técnicas, la verificación de los criterios de funcionamiento, la definición de la periodicidad de la calibración, la definición de errores máximos permitidos de acuerdo al fabricante o a las normas, la garantía de que las mediciones se realicen con patrones trazables, el ingreso de los equipos a un plan de mantenimiento preventivo como estrategia de seguimiento estricto del funcionamiento técnico y ensayos de seguridad eléctrica ${ }^{[8]}$. 
Un ejemplo de información sobre procesos de evaluación técnica de equipos correspondiente al primer nivel de evaluación o evaluación regulatoria es el que se presenta en el estudio de Revilla y cols. relativo al análisis de la normatividad sobre el registro y permiso de venta de equipos médicos en Argentina, Brasil, México y Uruguay, en el apartado "Certificación de Equipos y su calibración”.

En este estudio se analiza si en las normas de dichos países se incluyen o no acciones orientadas a dicha certificación y calibración (entendiendo a la calibración como una operación que establece una relación entre los valores y sus incertidumbres de medición asociadas). Encuentran que en Argentina existe una norma que establece que un grupo técnico deberá dar seguimiento a empresas, fabricantes e importadores de equipos biomédicos y tomará decisiones respecto a la situación encontrada; que en México existe una norma que establece que un responsable sanitario, jefe de servicio o comité intrahospitalario deberán de verificar la existencia de manuales de operación, de buenas prácticas de uso de equipos biomédicos, así como accesorios para su funcionamiento y capacitación del personal y que en Uruguay existirá un director técnico responsable que deberá de evaluar y conocer los productos que comercializa la empresa, capacitar al personal y brindar asesoría técnica a usuarios ${ }^{[35]}$.

Otros ejemplos de evaluación técnica en la etapa de utilización (N5), son los que se llevan a cabo para inspección de equipo médico identificando la causa de falla, con base en la organización, análisis de fallas y riesgo, como estrategias de mantenimiento ${ }^{[36]}$; para la evaluación de desempeño de equipos médicos considerando su estado, las recomendaciones del fabricante, el estado de los accesorios, aspectos de seguridad eléctrica, las pruebas específicas según el tipo de equipo, el modelo y el rango de operación, de acuerdo a sus especificaciones técnicas (p. ej. funcionalidad de la batería y de las alarmas, en su caso) ${ }^{[17]}$; o bien consi- derando la función que cumple, riesgo asociado a la aplicación clínica, antecedentes de reparación, requisitos de mantenimiento según fabricante ${ }^{[37]}$.

Los criterios y elementos considerados en propuestas de evaluación técnica a equipos o dispositivos médicos específicos serán por consiguiente también muy específicos, como los que se consideraron para probar un software desarrollado para automatizar las pruebas paramétricas para los estimuladores somatosensorial y auditivo de un equipo de potenciales evocados y evaluación del sistema neuromuscular ${ }^{[38]}$; para estudiar la resolución del contraste para mejorar la calidad de la

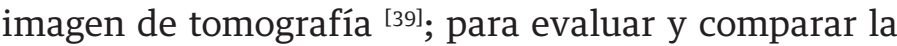
calidad de imágenes mamográficas en distintos centros de salud ${ }^{[40]}$; para analizar etiquetas implantadas con sistemas de identificación en radiofrecuencia (RFID) en animales y seres humanos [41]; para validar y analizar la confiabilidad de un medidor de impedancia para la evaluación de las terapias de rehabilitación neuromuscular ${ }^{[42]}$ y para validar la interpretación que entrega el electrocardiógrafo Cardiocid D sobre el trazo electrocardiográfico, en comparación con la del médico especialista ${ }^{[43]}$; el sistema para evaluar los transformadores de la fuente de poder de los equipos médicos [44]; la evaluación de la calidad de los instrumentos empleados en cirugía robótica ${ }^{[45]}$; la evaluación de las propiedades biomecánicas de la interfaz que rodea al implante dental ${ }^{[46]}$.

Finalmente, a manera de ejemplos de evaluación técnica para la toma de decisiones sobre sustitución de equipo (N6) se pueden mencionar los que presentan Mora y cols., en los que se propone un indicador para evaluar los aspectos técnicos de equipo médico para la toma de decisiones sobre el tiempo para sustituirlo. Los elementos que se toman en cuenta para llevar a cabo la evaluación técnica en este caso son los consumibles y las refacciones disponibles en cinco años, los años que el equipo tiene en operación, los días fuera de servicio, la función del equipo, la frecuencia de fallas, 
el riesgo físico y los requisitos de mantenimiento ${ }^{[47][48]}$. De manera similar, Ruiz utiliza cuatro criterios para la evaluación de la condición técnica de los dispositivos para decidir sobre su disposición final: la edad efectiva, refiriéndose al tiempo transcurrido desde que inicia su operación, medida en años; el grado de obsolescencia, definida como el soporte suministrado por el fabricante o proveedor del equipo a través de distribuidores o representantes locales o internacionales; la demanda, que hace referencia a la frecuencia de uso del equipo, considerada debido a la relación directa entre la demanda y la probabilidad de falla y por lo tanto al acercamiento al final de su vida útil y el estado de funcionamiento basado en los reportes de inspecciones realizadas por el personal de mantenimiento así como en los certificados de calibración ${ }^{[49]}$.

\section{Impactos éticos, sociales, legales o psicológicos}

Los impactos sociales de la tecnología médica también se llegan a mencionar cuando se habla de ETES. Algunos elementos de evaluación de los impactos éticos, sociales o legales a considerar podrían girar en torno a las disposiciones legales que afectan la adquisición, la accesibilidad de la tecnología, el cumplimiento de los derechos humanos, la integridad y dignidad humana, la equidad, los posibles conflictos con convicciones religiosas y culturales, la estigmatización y discriminación u otros factores como el de la necesidad de contar con la firma de consentimiento informado para situaciones especiales ${ }^{[3]}[4]$ [6] [13] [15] [22] [23] [50] [51].

Sin embargo, el nivel de importancia que se le da a los aspectos éticos, legales, sociales y psicológicos no se compara con el que se le da a los de seguridad, a los clínicos, técnicos, económicos o incluso a los institucionales, como los relacionados con las necesidades, encontrándose que este tipo de elementos de evaluación suelen ocupar el lugar menos preponderante en una ETES. Ardila y cols,, por ejemplo, en un estudio que realizan con el propósito de identificar las etapas críticas y los pasos a seguir en los procesos de incorporación de tecnología, identifican que para un grupo de profesionales en Ingeniería Clínica los aspectos éticos y legales ocupan el último nivel de importancia como criterios de evaluación ${ }^{[13]}$.

\section{Impacto organizacional}

La incorporación de nueva tecnología a una institución de atención a la salud puede ocasionar algún tipo de impacto desde el punto de vista organizacional, por lo que resulta conveniente considerar este aspecto dentro de los procesos de ETS, sobre todo en aquellos ubicados en los niveles de evaluación en hospitales y para la adquisición de equipo (N3 y N4). En este sentido Carvajal y cols. mencionan este aspecto como parte de su propuesta metodológica para la adquisición de tecnología biomédica, indicando que el DIB en colaboración con los departamentos administrativo y financiero, debería participar en este proceso enfocándose en la labor de gestión de la evidencia apropiada sobre su entorno organizacional ${ }^{[14]}$. En otro estudio, Chavarría y cols. presentan una herramienta de ETES para la selección y adquisición de equipos médicos (N4). Una de las etapas del desarrollo de la herramienta consistió en implementar el módulo para evaluar el impacto organizacional pidiéndole al usuario que calificara del uno al cinco (donde 1 significaba muchas repercusiones o cambios en la estructura de la organización al incorporar la tecnología y 5 significaba pocas repercusiones o cambios ${ }^{[15]}$. De manera similar, Gomez y cols. mencionan que la introducción de nuevos modos de resolver problemas diagnósticos y terapéuticos, en algunas ocasiones conlleva a cambios en la estructura, organización y necesidades cuantitativas y cualitativas de personal y que resulta una tarea obligada el evaluar el impacto para prever los cambios, además, reconocen que se tiene que seguir investigando sobre el impacto real de la ETES para el uso de la evidencia científica y apoyar las prácticas clínicas y la toma de decisiones de gestión en hospitales ${ }^{[3]}$. 
La interpretación de las definiciones y conceptos relacionados con los diferentes aspectos considerados en la evaluación varían entre países e instancias involucradas en una ETES, ocasionando que los elementos utilizados sean también muy diversos.

Quedan abiertas las preguntas para las ETES en Latinoamérica ¿Están representados todos los niveles de evaluación en la clasificación aquí propuesta (N1 a N6)? ¿Qué niveles se analizan en mayor medida? ¿Qué aspectos se analizan en cada nivel de evaluación y en qué proporción? ¿Qué elementos se analizan dentro de cada aspecto evaluado y en qué proporción? Suelen presentarse propuestas metodológicas para ETES pero en muchos casos no son claros los elementos utilizados para cada tipo de aspecto evaluado. Sería deseable contar con información sustentada en ejemplos ilustrativos de cada elemento para cada aspecto en los diferentes niveles de evaluación y sobre todo con el análisis del impacto que cada uno de los procedimientos, aspectos y elementos de evaluación propuestos ha tenido con respecto al alacance de los objetivos.

\section{¿CÓMO SE EVALÚA? MÉTODOS DE LA EVALUACIÓN DE TECNOLOGÍAS EN SALUD}

Para que una evaluación tenga validez y cumpla con el objetivo de determinar el valor del producto bajo escrutinio, se requiere de una metodología que refleje la manera en que se organizó tanto la información como los datos recabados. De esta forma se le da sustento a los resultados y robustez a las salidas del proceso.

Durante mucho tiempo la ETES en América Latina se ha hecho de manera empírica, fundamentalmente, debido a la falta de información y de datos sistematizados que permitan la aplicación de alguna metodología. Esto se muestra claramente al analizar diversos trabajos en los que se hace evaluación para la adquisición de equipo médico. En éstos se hace un análisis comparativo cualitativo de diversa información proporcionada por los proveedores y, conforme a los recursos financieros disponibles del establecimiento de salud en cuestión y la experiencia del tomador de decisión, se elige la "mejor" tecnología [14] [15]. En otros trabajos relacionados con la evaluación de tecnología médica instalada en los hospitales, se muestran intentos por sistematizar e integrar datos sobre los aspectos técnico, clínico y económico de los equipos médicos. A partir de la definición de variables, la asignación de factores de relevancia y la generación de alguna función matemática sencilla, se busca obtener información sobre el estado funcional del dispositivo médico ${ }^{[16]}$. También se ha desarrollado la evaluación de equipo médico que se utiliza en el hogar o en el medio laboral con metodologías propias de las ciencias sociales como la grounded theory que colecta información de naturaleza cualitativa, mientras que no se aprecia metodología alguna en su procesamiento ${ }^{[52]}$. Se observa que varias publicaciones han examinado el papel de la ETES a través de la recopilación de datos, pero aún existe un conocimiento limitado de cómo los tomadores de decisiones usan y valoran esta evidencia, así como el desafío de incorporar criterios sistematizados de manera explícita.

En los últimos 20 años ha surgido un gran interés en la aplicación de métodos que permitan la formalización del conocimiento sobre las tecnologías en salud disponibles en los diferentes establecimientos sanitarios. Algunos de estos trabajos incorporan la metodología six sigma y el análisis de decisiones multicriterio (MCDA por sus siglas en inglés) que han surgido como herramientas útiles para apoyar la toma de decisiones sobre la tecnología médica en la atención a la salud. A continuación se exponen algunas aplicaciones de estos dos métodos a la ETES.

\section{Método Six Sigma}

Six Sigma es una metodología de gestión de calidad centrada en el control de procesos cuyo objetivo es disminuir el número de defectos en la entrega de un 
producto o un servicio al cliente. Esta metodología, permite a las organizaciones, mejorar la capacidad de sus procesos incrementando su desempeño y disminuyendo los defectos. El nombre Six Sigma está relacionado con la letra griega sigma $(\sigma)$ que es utilizada en estadística para denominar la desviación estándar. Es la medida de la dispersión del área bajo la curva de distribución normal y mientras más alto sea el coeficiente sigma y por ende, menor la desviación estándar, el proceso es mejor, más preciso y menos variable. En estadística el valor de seis sigma corresponde a 3.4 defectos por millón. Para reducir los errores en el área de servicios se habla de un nivel entre 1 y 2 sigma ${ }^{[53]}$. De manera particular, la mayoría de los hospitales está trabajando con un nivel de 3 o 4 sigma ${ }^{[54]}$.

La metodología Six Sigma se ha empezado a utilizar en la evaluación de tecnologías para la salud con éxito en países de América Latina. En México, se ha aplicado en la reingeniería de procesos de atención a la salud donde se incorporan nuevas tecnologías, como es el caso de la innovación de procesos en el Departamento de Imagenología del Instituto Nacional de Enfermedades Respiratorias (INER por sus siglas en español), debido a la incorporación de un Sistema de Comunicación de Archivo de Imágenes (PACS por sus siglas en inglés) que tuvo gran impacto en la forma de atender a los pacientes y en la dinámica de trabajo del personal [55] [56] [57]. Posteriormente, en este mismo Instituto, la metodología se aplicó para mejorar el proceso de requisición, adquisición y dispensación de medicamentos ${ }^{[58]}$. Asimismo se aplicó en la mejora del proceso de gestión de la tecnología médica en los Servicios de Salud del Estado de Morelos ${ }^{[59]}$. En todos los casos, se obtuvo una mejora del proceso particular que se estudió, lo que prueba que la incorporación de métodos que formalizan el conocimiento relacionado con las tecnologías para la salud, es una estrategia para obtener resultados basados en evidencia que impactan en la atención a los pacientes y permiten demostrar la utilidad de la ingeniería biomédica en el medio hospitalario.

\section{Método de Decisión Multicriterio}

El Análisis de Decisión Multicriterio (MCDA, por su siglas en inglés) apoya la toma de decisiones dividiendo los problemas complejos en múltiples componentes y recurriendo a enfoques tanto cualitativos como cuantitativos para medirlos y posteriormente combinarlos ${ }^{[60]}$. Se ha encontrado que este método, aplicado a la ETES, proporciona información relevante que sirve de sustento a la toma de la decisión. Por ejemplo, en 2014 se llevó a cabo un trabajo cuyo objetivo fue desarrollar un indicador para estimar el acceso a los servicios de diagnóstico por imágenes médicas (IM) en hospitales del Sistema de Salud Pública de Costa Rica. Tomando en cuenta los aspectos de infraestructura, capacidad tecnológica, epidemiológicos, demográficos y de ubicación con relación a la Capital, se definieron 14 variables y 7 indicadores.

Estos indicadores se integraron en una función matemática que a su vez dio origen a un indicador global que refleja el nivel de acceso de la población al servicio por IM correspondiente a su región geográfica de influencia. Al aplicar dicho indicador global a las 8 subredes con regiones geográficas definidas dentro del territorio de Costa Rica se encontró que en 3 de ellas se presentaba un acceso deficiente, en otras 3 el acceso era regular y sólo en 2 subredes se contaba con un acceso bueno. Al encontrar que el acceso no era equitativo en cada subred, era posible trabajar en propuestas de gestión tecnológica que fortalecieran los servicios de IM regionales, contribuyendo a descentralizarlos de los Hospitales Generales ubicados en la capital [61] .

Otro esfuerzo en esta dirección fue un desarrollo realizado en México, en el que se elaboró una herramienta para evaluar los aspectos técnico, clínico y económico del equipo médico y priorizar su sustitución en el Instituto Nacional de Pediatría [47]. Por otro lado, en Colombia se desarrolló un trabajo para demostrar el potencial del uso de MCDA en las decisiones de asignación de recursos en el sistema de salud. Mediante la 
herramienta denominada EVIDEM (Evidence and Value: Impact on Decision-Making), se realizó un estudio piloto para evaluar 4 fármacos para atender la hemofilia severa, la prevención de la diarrea, el desarrollo de una terapia hormonal para mujeres en la postmenopausia con cáncer de mama y el riesgo cardiovascular moderado a alto. Los resultados que se obtuvieron fueron un indicador de la diversidad presente en cuanto a las perspectivas e interpretación de la evidencia presentada a los que participaron en la evaluación. Se concluyó que el uso de EVIDEM para asistir la toma de decisiones con respecto a la asignación de recursos en Colombia se podía tomar como un medio para incorporar la ETES en dicho proceso y para priorizar las intervenciones en salud ${ }^{[62]}$.

De los trabajos presentados se resalta la importancia del desarrollo de metodologías para la ETES. Los resultados que se obtienen de un proceso basado en métodos claros y precisos fortalecen la evidencia obtenida, misma que sustenta las conclusiones que sirven de fundamento a la toma de decisiones. En la medida en que se desarrollen ETES con mayor rigor metodológico, se podrá tener mayor confianza en la calidad de las salidas, impactando de manera positiva las acciones que se lleven a cabo en consecuencia.

\section{¿PARA QUÉ SE EVALÚA? EVALUACIÓN DE TECNOLOGÍAS EN SALUD Y LA GENERACIÓN DE POLÍTICAS PÚBLICAS}

De acuerdo a la definición de evaluación de tecnologías en salud de la OMS, su propósito es contribuir “al proceso de formulación de políticas y a la toma de decisiones con fundamento, especialmente para los países en desarrollo y emergentes" [63]. En este sentido, resulta relevante observar la correlación entre los resultados obtenidos por la evaluación de tecnologías en salud y la elaboración, implementación, monitoreo y evaluación de políticas en esta materia. A fin de analizar el impacto que se ha tenido en la generación de políticas en la adopción, utilización y destino final de las tecnologías en salud en América Latina, es conveniente plantear un marco teórico general que sirva de base.

Una política puede expresarse como "el curso de acción que sigue un actor o un conjunto de actores al tratar un problema o asunto de interés" [64]; y una política pública puede definirse como una intervención deliberada del Estado cuyo objetivo es corregir o modificar una situación de naturaleza social o económica y que ha sido reconocida como un problema público ${ }^{[65]}$.

Para analizar una política pública, Molina et.al ${ }^{[66]}$ se basan en Hogwood y Gunn para presentar cuatro dimensiones de enfoque:

Contenido: estudia cómo surge y se desarrolla una política pública, así como los resutados que genera.

Proceso: estudia las etapas por las que pasa la política pública, evaluando la influencia que tienen sobre ella los factores involucrados.

Evaluación: estudia los resultados e impactos que se producen al poner en marcha la política pública. Esta se puede enfocar en las causas, las cuales se orientan hacia la política pública en sí misma o hacia la generación de la información requerida de un problema que sustente la política.

Soporte: estudia por un lado la mejora del sistema de información y del apoyo gubernamental, mientras que por otro lado se analizan las opciones influyendo en el proceso de la política.

El proceso para formular políticas públicas no siempre es claro. Lasswell ${ }^{[67]}$ propone un sistema "paso a paso" para elaborarlas y se asume que la claridad en los pasos a seguir es inmediata. Sin embargo, al realizar una política pública se evidencia que no es así. Algunos aspectos que sobresalen a este respecto son los siguientes: 
1. Dado que se trata de implementar acciones para la solución de problemas, la definición de éste debe ser muy precisa para que no ocurra el caso de que la salida del mismo sea efectivamente una solución para un grupo, pero implique un problema para otro.

2. Las etapas de elaboración e implementación presentan elementos que se pueden llegar a mezclar entre sí, dificultando el seguimento puntual de cada una de ellas.

3. El surgimiento de una política no siempre atiende una necesidad; algunas políticas nacen como consecuencia de la identificación de oportunidades más que de la respuesta a problemas.

Las políticas públicas en la práctica surgen como el resultado de la interacción de muchos actores, algunos con posturas contrarias. El hecho de que la mayoría de las políticas sean el resultado de una serie de acuerdos entre las diversas partes interesadas hace que el proceso de su generación sea complejo y, en muchas ocasiones, largo. Con respecto a la salud, y en particular en el ámbito de las tecnologías médicas, la generación de políticas públicas en América Latina ha presentado intentos por identificar problemáticas y oportunidades que las fundamenten.

Tomando como base la ETES, durante la última década se han desarrollado diversos proyectos de políticas públicas en los que se puede apreciar una tendencia a institucionalizar esta actividad a través de la creación de iniciativas como la Red de Evaluación de Tecnologías en Salud de las Américas (RedETSA), la Promoción de la Toma de Decisiones basada en la Evidencia para la Introducción de Vacunas Nuevas y Subutilizadas (ProVac) por parte de al Organización Panamericana de la Salud (OPS), los repositorios de información en este tema o las colaboraciones entre los países de la región ${ }^{[68]}$.
Una estrategia para determinar el propósito de la ETES es a través de la identificación de los principios de buenas prácticas en esta materia así como de las barreras que impiden su implementación. En 2016 se abordó el tema a partir de la celebración de Primer Foro Latinoamericano de Políticas de Evaluación de Tecnologías Sanitarias en San José Costa Rica, cuya intención fue determinar cuáles de los principios postualdos a nivel global podrían aplicarse al contexto de América Latina. Con base en el análisis de la relevancia en la mejora de los procesos de ETES y de las brechas más grandes entre el escenario ideal y el real, se determinó el primer conjunto de principios aplicables [69]: transparencia en los procesos de ETES, involucramiento de los actores relevantes, mecanismos de apelación, mecanismos para la priorización de los temas a evaluar y vínculo entre la evaluacion y la toma de la decisión. Hay un acuerdo en que estos principios deberán aplicarse atendiendo el contexto local, dados los diversos grados de avance que se tiene en los países de la región.

Un caso de estudio que resulta interesante es el que se desarrolló para establecer el uso sistemático de la evidencia para informar al tomador de decisiones, desembocando en la creación de la Agencia de ETES del gobiendo de Colombia. En este caso se utilizó el modelo de John Kingdon para desarrollar el proceso de análisis de políticas que generó la creación de la Agencia. El modelo de Kingdon se fundamenta en la teoria de las ventanas de oportunidad, la cual establece que deben coincidir tres corrientes en el tiempo para abrirlas: la corriente de los problemas, la de los acontecimientos políticos y la de las soluciones; la confluencia de estas corrientes define la agenda [70]. Retomando el caso, se puede apreciar cómo se abrieron dos ventanas de oportunidad en un segmento temporal de 10 años, y cómo los diferentes factores que se van incorporando al problema y a la solución definen los grupos de interés que interactúan para obtener como salida la Agencia de ETES. Asimismo se 
plantea que los obstáculos que se encuentran en el camino están altamente correlacionados con el contexto local tanto económico como político y social ${ }^{[30]}$.

En Argentina se reportó el caso de la creación de un programa que con base en la ETES, se aplicó a un hospital público especializado en Pediatría desde 2001. El programa se fundamentó en la realización de reportes de apoyo para la toma de decisiones en materia de adquisición de tecnología, elaboración e implementación de guías pediátricas, desarrollo de capacidades profesionales en investigación y gestión, y soporte técnico para proyectos de investigación de servicios de salud. En un periodo de diez años se realizaron reportes de ETES en fármacos, procedimientos terapéuticos, preventivos y diagnósticos, así como programas institucionales. Asimismo se cubrieron los ámbitos de efectividad, seguridad, impacto en los presupuestos, relación costo-efectividad e impacto organizacional. Dos aspectos relevantes de esta experiencia que se reportaron fueron la resistencia de las partes interesadas (stakeholders) a aceptar la utilidad de la toma de decisiones basada en la evidencia, la comunicación entre los investigadores y los practicantes en la clínica que genera variabilidad importante en la práctica. Quizá la mayor limitante reportada es que se contaba con solamente una persona de tiempo completo ${ }^{[71]}$.

Con el fin de que una política pueda ser concebida, e independientemente del modelo a utilizar, se requiere que la participación de los stakeholders sea activa y, como se mencionó anteriormente, se ha vuelto prioritaria su participación en materia de ETES. La situación en la región de Améric Latina refleja una participación parcial e informal en los procesos y en la toma de decisiones. En 2017 se celebró el Segundo Foro Latinoamericano de Políticas en ETES en Lima Perú, con la finalidad de acordar la manera de involucrar más a los stakeholders tanto en los marcos conceptuales como en los metodológicos. En esa ocasión el evento se enfocó en los grupos de stakeholders considerados como de la mayor prioridad en la región: pacientes y cuidadores, y los productores de tecnologías en salud. Como resultado de los trabajos desarrollados en el foro, se concluyó que, si bien la incorporación de los stakeholders clave es prioritario, la manera en cómo se haga dependerá del contexto de cada país ${ }^{[50]}$.

La institucionalización de la ETES no garantiza que se genere información enfocada a orientar las políticas resultantes. Es muy probable que una política encaminada a resolver un problema confronte intereses de diversas naturalezas, haciendo ganadores a unos grupos y perdedores a otros. El conflicto de intereses, los tiempos para agendar, desarrollar, monitorear y evaluar dichas políticas, así como la planeación y sobre todo los contextos locales, hacen necesario que se involucren más actores en la ETES. Bajo este marco de referencia las instituciones adquieren un papel coordinador importante para organizar las acciones diirigidas a atender los problemas o situaciones que éstas pueden detectar con mayor precisión.

Dos aspectos relevantes a analizar es la baja producción de publicaciones en esta materia y que provengan, esencialmente, del mismo grupo de trabajo. Constrastando con lo reportado en cuanto al análisis y los métodos, se aprecia una necesidad por desarrollar más investigación en integrar la ETES a la toma de decisiones. Asimismo, como se reportó en los casos de Colombia y sobre todo Argentina, es notable cómo la magnitud de las funciones a realizar en ETES recae en un mínimo de recurso humano, el cual no logra cubrir todos los aspectos que forman parte de esta función.

\section{CONCLUSIONES}

En este artículo se abordaron tres perspectivas de la evaluación de tecnologías de salud (ETES): qué elementos se evalúan, cómo se hace la evaluación y para qué se realiza una ETES así como su influencia en el desarrollo de políticas públicas. 
Primeramente se expusieron las perspectivas que pueden conducir una ETES. A nivel macro se sitúan las evaluaciones en materia regulatoria y para el desarrollo de políticas públicas de salud; a nivel micro aquellas que se llevan a cabo en los establecimientos de salud para evaluar tanto la adquisición y la incorporación de equipo médico, así como la tecnología médica instalada y su disposición final.

Sobre los aspectos que se evalúan en las tecnologías de salud, se identificaron: seguridad, eficacia, efectividad y eficiencia, así como los económico-financieros, clínicos y técnicos. Estos últimos son los más desarrollados, debido, probablemente, a que los parámetros a evaluar quedan establecidos por el fabricante y se miden directamente en el equipo médico. No así el aspecto clínico, donde los datos y la información deben adquirirse a partir de la interacción con el usuario de la tecnología, es decir, con el personal médico y paramédico. En ocasiones se evalúa el impacto ético, social, legal, psicológico y/u organizacional de la tecnología médica aunque con mucho menor importancia, ya que la adquisición de conocimiento sobre estos aspectos es altamente subjetiva y difícil de formalizar.

Con respecto a cómo se realiza la ETES, en la mayoría de los trabajos revisados se exponen estrategias empíricas para el análisis de la información. La toma de decisiones depende, en buena medida, de la experiencia de quien lo hace. Sin embargo, se empiezan a visualizar algunos estudios en los que se emplean métodos que permiten formalizar el conocimiento de la ETES, como Six Sigma y el Método de Decisión Multicriterio, con los que se comprueba que el empleo de métodos formales proporciona evidencia clara y objetiva de las tecnología, útil para tomar alguna decisión de interés para el establecimiento de salud en cuestión.

Por otro lado, la generación de políticas públicas en salud relacionadas con la ETES en los países de América
Latina es incipiente, apenas se están identificando las problemáticas nacionales y las estrategias a seguir para su solución. Esto se ha hecho mediante la creación de instancias de ETES nacionales y/o regionales, así como con la edición de dos Foros Latinonamericanos de Políticas de Evaluación de Tecnología Sanitaria realizados en San José en 2016 y en Lima en 2017.

Finalmente, queda claro para todos los países de América Latina el valor de la ETES en la toma de decisiones en materia de los servicios de salud y que a pesar de los esfuerzos realizados, no se han logrado los avances esperados. En 2014 los miembros de la OMS aprobaron una resolución sobre la ETES y la cobertura universal en salud. Una de las primeras directrices fue "considerar el establecimiento de sistemas nacionales de intervención en salud y evaluación de tecnología, alentando la utilización sistemática de [...] evaluación de tecnología en apoyo de la cobertura de salud universal para informar las decisiones de políticas" [72]. Los esfuerzos realizados para establecer las bases sobre las que se construyan tanto los principios de aplicar la ETES como las organizaciones que los implementen en América Latina han surgido principalmente a raiz de esta resolución. Los foros de discusión sobre el tema han servido para poner de acuerdo a los participantes en lo que se deben enfocar al momento de desarrollar la ETES y de integrarla a la toma de decisiones, pero no se observa una clara estrategia que efectivamente permita una vinculación robusta entre las dos funciones, tanto a nivel macro, como a nivel micro. Es claro entonces, que los países de América Latina deben seguir trabajando en el desarrollo de políticas, estrategias y métodos de evaluación de tecnologías en salud, que permitan en un futuro cercano lograr que las instituciones que proveen servicios de salud tengan disponibles las tecnologías que respondan a los problemas epidemiológicos nacionales y atiendan las necesidades de diagnóstico, tratamiento y rehabilitación de la población de cada país. 


\section{REFERENCIAS}

[1] WHO | HTA Definitions [Internet]. Who.int. 2019

[citado 2019 Jun 4]. Disponible en: https://www.who.int/healthtechnology-assessment/about/Defining/en/

[2] Barrenechea Loo M. Desarrollo de la evaluación de tecnologías en salud en algunos países de Latinoamérica. Revista Cubana de Salud Pública [Internet]. 2017 Dic [citado 2019 Jun 04] ; 43(4). Disponible en: $h t t p: / / s c i e l o . s l d . c u / s c i e l o . p h p ? s c r i p t=s c i$ arttext\&pid $=$ S0864-34662017000400010\&lng=es

[3] Barrientos Gómez J, Marín Castro A, Becerra Ruiz L, Tobón Arango M. La evaluación de nuevas tecnologías en salud en hospitales: revisión narrativa. Medicina U.P.B. [S.1] [Internet]. 2017 Feb [citado 2019 Jun 4]; 35(2): 120-134. Disponible en: https://revistas.upb.edu. co/index.php/Medicina/article/view/7146.

DOI: http://dx.doi.org/10.18566/medupb.v35n2.a06

[4] Manterola Carlos, Otzen Tamara, Castro Mayra, Grande Luis. Health Technology Assessment (HTA). An Overview of the Concept and its Scope. International Journal of Morphology. [Internet]. 2018 Sep [citado 2019 Jun 05]; 36(3): 1134-1142. Disponible en: https://scielo.conicyt.cl/scielo.php?script=sci_arttext\&pid=S071795022018000301134\&lng=en. DOI: http://dx.doi.org/10.4067/ S0717-95022018000301134

[5] Martelli N, Hansen P, van den Brink H, Boudard A, Cordonnier A, Devaux $\mathrm{C}$ et al. Combining multi-criteria decision analysis and mini-health technology assessment: A funding decision-support tool for medical devices in a university hospital setting. Journal of Biomedical Informatics. 2016;59:201-208. DOI: https://doi. org/10.1016/j.jbi.2015.12.002

[6] Pichon-Riviere A, Augustovski F, García Martí S, Sullivan SD, Drummond M. Transferability of Health Technology Assessment Reports in Latin America: An Exploratory Survey of Researchers and Decision Makers. International Journal of Technology Assessment in Health Care. Cambridge University Press; 2012;28(2):180-6. DOl: https://doi.org/10.1017/S0266462312000074

[7] Rabelo R, Canuto V, Petramale C, Mega T. OP133 Health Technology Assessment in Brazil: A 5-year Review of Brazilian Health System (CONITEC) Activities. International Journal of Technology Assessment in Health Care. Cambridge University Press; 2017;33(S1):62. DOI: https://doi.org/10.1017/S0266462317001921

[8] Ramírez Barrera A.F, Martínez G J.F, Hidalgo Vázquez E. Modelo de Gestión para la Aplicación del Control Metrológico Legal y la Evaluación de la Confromidad en Equipos Biomédicos. Revista Ingeniería Biomédica [Internet]. 2017 June [citado 2019 Jun 6] ;

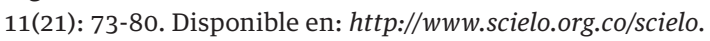
php?script=sci_arttext\&pid $=$ S1909-97622017000100010\&lng=en

[9] Ríos P, Rivera A, Oropeza I, Ramírez O. The Update of the Mexican Health Care Formulary and Supply Catalog in the Context of the Health Technology Assessment. Value in Health Regional Issues. 2014;5:29-34. DOI: https://doi.org/10.1016/j.vhri.2014.08.002

[10] Ferraz Marcos B, Soárez P Coelho de, Zucchi P. Health technology assessment in Brazil: what do healthcare system players think about it? Sao Paulo Medical Journal [Internet]. 2011 [citado 2019 Jun 05]; 129(4):198-205. Disponible en: http://www.scielo.br/scielo. php?script=sci_arttext\&pid $=$ S1516-31802011000400002\&lng $=$ en . DOI: http://dx.doi.org/10.1590/S1516-31802011000400002
[11] Ruiz Ibañez C, Franco A, Mejia Hoyos H, Fajardo R. Perspectivas en seguridad del paciente. Entrevista con líderes colombianos. Revista Ingeniería Biomédica. [Internet]. 2010 Dec [citado 2019 Jun 04];

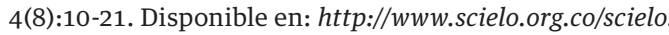
php?script=sci_arttext\&pid=S1909-97622010000200002\&lng=en .

[12] Fors López M, Peña Amador D. Algunas consideraciones generales sobre el desarrollo de la evaluación de productos farmacéuticos y biotecnológicos y equipos médicos en cuba y el mundo. Revista Cubana de Investigaciones Biomédicas [Internet]. 1999 Ago [citado 2019 Jun 05]; 18(2):117-120. Disponible en: http://scielo. sld.cu/scielo.php?script=sci_arttext\&pid=S086403001999000200008\&lng=es

[13] Ardila M, Gómez A, Camacho-Cogollo J. Método de incorporación estratégica de tecnología biomédica para instituciones de salud. Revista Ingeniería Biomédica. 2017;10(20). DOI: https://doi.org/10.24050/19099762.n20.2017.1071

[14] Carvajal Tejada M, Ruiz Ibañez C. Evaluación Técnica y Clínica de Tecnología Biomédica en Procesos de Adquisición: un Enfoque en Evaluación de Tecnologías en Salud. Revista Ingeniería Biomédica [Internet]. 2008 Dec [citado 2019 Jun 07] ; 2(4): 34-45. Disponible en: $h t t p: / / w w w . s c i e l o . o r g . c o / s c i e l o . p h p ? s c r i p t=s c i_{-}$ arttext\&pid=S1909-97622008000200006\&lng=en .

[15] Chavarría T, Molina T. Herramienta de Evaluación de Tecnologías para la Adquisición de Equipos Biomédicos. Revista Ingeniería Biomédica. [Internet]. 2017 June [citado 2019 Jun 09]; 11(21): 13-19. Disponible en: http://www.scielo.org.co/scielo.php?script=sci arttext\&pid $=$ S1909-97622017000100002\&lng $=e n$.

[16] Duque M, Lores C. Diseño y Aplicación de un Protocolo de Evaluación de Equipos Médicos. In: Folgueras Méndez J et al. (eds). V Latin American Congress on Biomedical Engineering CLAIB 2011. Springer Berlin Heidelberg: IFMBE Proceedings; 2013 [citado 2019 Jun 8]. 33:417-420.

DOI: https://doi.org/10.1007/978-3-642-21198-0_107

[17] Correa M, Villalba M, García J. Protocolos para evaluación de desempeño en equipos médicos. Revista Ingeniería Biomédica. 2017;11(22):65-71.

DOI: https://doi.org/10.24050/19099762.n22.2017.1185

[18] Acevedo J., Saldarriaga N., Orozco J S., García J H. Design of a Validation Protocol for Medical Technology According to Current Standards. In: Torres I., Bustamante J., Sierra D. (eds) VII Latin American Congress on Biomedical Engineering CLAIB 2016. Springer, Singapore: IFMBE Proceedings; 2017[citado 2019 Jun 8]. 60: 634-637. DOI: https://doi.org/10.1007/978-981-10-4086-3 159

[19] González Vargas A, Sánchez Benavides A, Betancourt Hernández A, Mantilla Ramirez C. Technovigilance and risk management as tools to improve patient safety in Colombian health care institutions. Revista Ingeniería Biomédica. 2017;11(21). DOI: https://doi.org/10.24050/19099762.n21.2017.1173

[20] Arregui M, Alfaro N, Baldizzoni M., Wald I., Gambogi R., Ferreira A., Simini F. Reducción del Riesgo en Equipos Biomédicos y en Instalaciones Eléctricas de Entornos Clínicos. Revista Mexicana de Ingeniería Biomedica. 2019; 40(1):1-13.

DOI: https://doi.org/10.17488/RIIIB.40.1.3. 
[21] Espinoza MA, Cabieses B. Equity in Health and Health Technology Assessment in Chile. Revista Médica de Chile [Internet]. 2014 Jan [citado 2019 Jun 14]; 142( Suppl 1 ):45-49. Disponible en: https:// scielo.conicyt.cl/scielo.php?script=sci_arttext\&pid=S003498872014001300008\&lng=en. http://dx.doi.org/10.4067/So03498872014001300008 DOI: https://dx.doi.org/10.4067/S0034-98872014001300008

[22] Margotti A, Ferreira F, Santos F, Garcia R. Health technology assessment to improve the medical equipment life cycle management. 35th Annual International Conference of the IEEE Engineering in Medicine and Biology Society (EMBC). IEEE; Osaka 2013. p. 354-357. DOI: https://doi.org/10.1109/EMBC.2013.66095`10

[23] Arellano L, Reza M, Blasco J, Andradas E. A content analysis of health technology assessment programs in Latin America. International Journal of Technology Assessment in Health Care. 2009;25(4):570-576. DOI: https://doi.org/10.1017/S026646230999033X

[24] Lingg M, Mansilla A, Durán-Arenas L, Wyss K. The regulation, assessment, and management of medical devices in Mexico: How do they shape the quality of delivered healthcare? Safety in Health. 2017;3(1):3-4. DOI: https://doi.org/10.1186/s40886-017-0055-8

[25] Marsh K, Sculpher M, Caro J, Tervonen T. The Use of MCDA in HTA: Great Potential, but More Effort Needed. Value in Health. 2018;21(4):394-397.

DOI: https://doi.org/10.1016/j.jval.2017.10.001

[26] López Bastida J, Oliva J, Antoñanzas F, García-Altés A, Gisbert R, Mar Javier et al . Propuesta de guía para la evaluación económica aplicada a las tecnologías sanitarias. Gac Sanit [Internet]. 2010 Abr [citado $2019 \mathrm{Jul} \mathrm{06]} \mathrm{;} \mathrm{24(} 2$ ): 154-170. Disponible en: http:// scielo.isciii.es/scielo.php?script=sci_arttext\&pid=S0213$91112010000200012 \& \operatorname{lng}=e s$.

[27] Gómez M., Villalta Y.H., Suen J.E., González R.I. Plastic Casing in Medical Equipment: Evaluation of 3D Design and Molding Simulation. In: Torres I., Bustamante J., Sierra D. (eds) VII Latin American Congress on Biomedical Engineering CLAIB 2016. Springer, Singapore: IFMBE Proceedings; 2017[citado 2019 Jun 16]. 60: 118-121. DOI: https://doi.org/10.1007/978-981-10-4086-3_30

[28] Gold M, Siegel J, Russell L, Weinstein M. Cost-effectiveness in health and medicine. New York: Oxford University; 1996.

[29] Beckerman R, Chowdhury C, Park S. Guardians of opportunity: emerging health technology assessment in Brazil, South Korea and Taiwan. Journal of Pharmaceutical Health Services Research. 2013;4(3):125-129. DOI: https://doi.org/10.1111/jphs.12020

[30] Castro H. Advancing HTA in Latin America: The Policy Process of Setting up an HTA Agency in Colombia. Global Policy. 2017;8:97102. DOI: https://doi.org/10.1111/1758-5899.12333

[31] Lemmer T, Piedade A, de Oliveira R, Moreira E, Julian G. Health Technology Assessment In Brazil: Metrics From The Reports Of The National Commission For Incorporation Of Technologies (Conitec). Value in Health. 2016;19(7):A449-A450. DOI: https://doi.org/10.1016/j.jval.2016.09.597

[32] Lingg M, Merida-Herrera E, Wyss K, Durán-Arenas L. Attitudes of Orthopedic Specialists Toward Effects of Medical Device Purchasing. International Journal of Technology Assessment in Health Care. Cambridge University Press; 2017;33(1):46-53. DOI: https://doi.org/10.1017/S0266462317000101
[33] Avendaño G, Rienzo A, Danyau L. Importance of HTA in modern Clinical Engineering. In Eskola H., Väisänen O., Viik J., Hyttinen J. (eds) EMBEC \& NBC 2017. Springer, Singapore: IFMBE Proceedings; 2017 [citado 2019 Jun 14]. 65:73-76. DOI: https://doi.org/10.1007/978-981-10-5122-7_19

[34] Cabrera P R, Gutiérrez N A. Estudio de la Usabilidad de Dispositivos Médicos Mediante la Investigación del Factor Humano: Caso Monitores de Glicemia. In: Folgueras Méndez J et al. (eds). V Latin American Congress on Biomedical Engineering CLAIB 2011. Springer Berlin Heidelberg: IFMBE Proceedings; 2013 [citado 2019 Jun 14]. 33:417-420. DOI: https://doi.org/10.1007/978-3-642-21198-0_108

[35] Revilla G, Borba F, Taborda R, Simini F. Comparación de normas para el registro y permiso de venta de equipos biomédicos en Argentina, Brasil, México y Uruguay. XLI Congreso Nacional De Ingeniería Biomédica [Internet]. 2018 [citado 2019 Jun 16]. 5:543546. Disponible en: https://memorias.somib.org.mx/index.php/ memorias/article/view/718

[36] Coronel Mendoza, E., Segura Pérez, E. Metodología para la inspección de equipo médico. XLI Congreso Nacional De Ingeniería Biomédica [Internet]. 2018 [citado 2019 Jun 16]. 5:534-537. Disponible en: $h t t p: / / m e m o r i a s . s o m i b . o r g . m x / i n d e x . p h p / m e m o r i a s /$ article/view/682

[37] Enriquez L, Correa M, Garcia J, Alvarez L. Development and application of a performance evaluation model in medical devices. 2018 Global Medical Engineering Physics Exchanges/Pan American Health Care Exchanges (GMEPE/PAHCE). 2018;:1-6. DOl: https://doi.org/10.1109/GMEPE-PAHCE.2018.8400753

[38] Crespo-Ruiz A., Chang-Duany J., Chailloux-Peguero J. Automatización de las Pruebas Paramétricas para los Estimuladores Somatosensorial y Auditivo del Equipo Neurónica 5I. In: Folgueras Méndez J et al. (eds). V Latin American Congress on Biomedical Engineering CLAIB 2011. Springer Berlin Heidelberg: IFMBE Proceedings; 2013 [citado 2019 Jun 15]. 33:436-439. DOI: https://doi.org/10.1007/978-3-642-21198-0_112

[39] Lataza O., Pérez M., Lorenzo J.V., Taboada A., Orozco M. Estudio de Resolución de Contraste en Tomografía Computarizada Multicorte. In: Folgueras Méndez J et al. (eds). V Latin American Congress on Biomedical Engineering CLAIB 2011. Springer Berlin Heidelberg: IFMBE Proceedings; 2013 [citado 2019 Jun 15]. 33:397-400. DOI: https://doi.org/10.1007/978-3-642-21198-0_102

[40] Morales M.M., Camargo M.L., Romo R., Graffigna J.P. Estudio Multicéntrico para el Análisis de Calidad de Imagen de Equipos Mamográficos con Tecnología Convencional. In: Folgueras Méndez $\mathrm{J}$ et al. (eds). V Latin American Congress on Biomedical Engineering CLAIB 2011. Springer Berlin Heidelberg: IFMBE Proceedings; 2013 [citado 2019 Jun 15]. 33:425-428. DOI: https://doi.org/10.1007/978-3-642-21198-0_109

[41] Pirrone J., Huerta M., Clotet R., Gonzalez R. Análisis del Uso de Etiquetas RFID Implantables en Seres Vivos. In: Folgueras Méndez $\mathrm{J}$ et al. (eds). V Latin American Congress on Biomedical Engineering CLAIB 2011. Springer Berlin Heidelberg: IFMBE Proceedings; 2013 [citado 2019 Jun 15]. 33:440-443 DOI: https://doi.org/10.1007/978-3-642-21198-0_113

[42] Reta J.M., Manresa J.A.B., Tabernig C.B. Validity and Reliability of an Impedance Meter Device for the Assessment of Neuromuscular Rehabilitation Therapies. In: Braidot A., Hadad A. (eds). VI Latin American Congress on Biomedical Engineering CLAIB 2014. Springer Cham: IFMBE Proceedings; 2014 [citado 2019 Jun 16]. 49:956-959. DOI: https://doi.org/10.1007/978-3-319-13117-7_242 
[43] Varona M.M.R., Rubio J.R., Fernández R.G., Sigler A.F. Estudio Preliminar de Anomalías Electrocardiográficas. In: Folgueras Méndez J et al. (eds). V Latin American Congress on Biomedical Engineering CLAIB 2011. Springer Berlin Heidelberg: IFMBE Proceedings; 2013 [citado 2019 Jun 16]. 33:393-396. DOI: https://doi.org/10.1007/978-3-642-21198-0_101

[44] Rodriguez CJ, Oller CA, Bruni RG, Vanella OR, Am Taborda R. System for the evaluation of power supply transformers in medical equipment. Journal of Physics: Conference Series 2011 (Vol. 313, No. 1, p. 012002). IOP Publishing.

[45] Teneud L.J., Silva R.J. Evaluación de Calidad de los Instrumentos Empleados en Cirugía Robótica. In: Folgueras Méndez J et al. (eds). V Latin American Congress on Biomedical Engineering CLAIB 2011. Springer Berlin Heidelberg: IFMBE Proceedings; 2013 [citado 2019 Jun 14]. 33:452-455.

DOI: https://doi.org/10.1007/978-3-642-21198-0_116

[46] Vayron R., Haiat G. Assessment of the Biomechanical Properties of the Interface Surrounding a Dental Implant: An In Vitro Approach. In: Braidot A., Hadad A. (eds). VI Latin American Congress on Biomedical Engineering CLAIB 2014. Springer Cham: IFMBE Proceedings; 2014 [citado 2019 Jun 14]. 49:5-8. DOI: https://doi.org/10.1007/978-3-319-13117-7 2

[47] Mora-García T, Piña-Quintero F, Ortiz-Posadas M. Medical Equipment Replacement Prioritization Indicator Using Multicriteria Decision Analysis. Progress in Artificial Intelligence and Pattern Recognition. 2018;11047:271-279. DOI: https://doi.org/10.1007/978-3-030-01132-1 31

[48] Ortiz-Posadas, M., Mora-García, T., \& Piña-Quintero, M. Un Indicador de Evaluación Técnica para Determinar la Prioridad de Sustitución de Equipo Médico. XLI Congreso Nacional De Ingeniería Biomédica [Internet]. 2018 [citado 2019 Jun 17]. 5:526529. Disponible en: http://memorias.somib.org.mx/index.php/ memorias/article/view/540

[49] Ruiz Chaves Victor. Diseño de un manual de procedimientos para la evaluación y disposición final de la tecnología biomédica en la empresa tecnoclínica LTDA. Repositorio educativo digital. $h t t p: / /$ hdl.handle.net/10614/10861

[50] Pichon-Riviere A, Soto N, Augustovski F, Sampietro-Colom L. Stakeholder Involvement in the Health Technology Assessment Process in Latin America. International Journal of Technology Assessment in Health Care. 2018;34(3):248-253. DOI: https://doi.org/10.1017/S0266462318000302

[51] Ritrovato M, Faggiano F, Tedesco G, Derrico P. Decision-Oriented Health Technology Assessment: One Step Forward in Supporting the Decision-Making Process in Hospitals. Value in Health. 2015;18(4):505-511. DOI: https://doi.org/10.1016/j.jval.2015.02.002

[52] Laree D., Reyes P. Método para la evaluación del uso seguro de dispositivos médicos en el ambiente del hogar. Revista Ingeniería Biomédica [Internet]. 2013 [citado 2019 Jun 18] ; 7( 13 ): 43-56. Disponible en: $h t t p: / / w w w . s c i e l o . o r g . c o / s c i e l o . p h p ? s c r i p t=s c i$ arttext\&pid $=$ S1909-97622013000100005\&lng $=$ en\&nrm $=i s o$

[53] Thawani S. Six sigma-strategy for organizational excellence. Total Quality Management \& Business Excellence. 2004 Jul 1;15(56):655-64.

\section{DOI: https://doi.org/10.1080/14783360410001680143}

[54] Bhardwaj P., Power of Six Sigma in Hospital and Healthcare Management. [Internet]. Ind Medica. [citado 7 Jun 2008] Disponible en http://cyberlectures.indmedica.com/show/123/1/ Power_Of_Six_Sigma_In_Hospital_and_Healthcare_Management
[55] García-Porres J, Ortiz-Posadas MR, Pimentel-Aguilar AB: Lean Six Sigma Applied to a Process Innovation in a Mexican Health Institute's Imaging Department. 30th Annual International Conference of the IEEE Engineering in Medicine and Biology Society (EMBC). IEEE; Vancouver 2008. p 5125- 5128. DOI: https://doi.org/10.1109/IEMBS.2008.4650367

[56] García-Porres J., Ortiz-Posadas M.R. Overall Sigma Level of an Imaging Department through Process Innovation. In: Dössel O., Schlegel W.C. (eds). World Congress on Medical Physics and Biomedical Engineering 2009. Springer, Berlin, Heidelberg: IFMBE Proceedings; 2009 [citado 2019 Jun 18]. vol 25/5: 377-380. DOI: https://doi.org/10.1007/978-3-642-03904-1_105

[57] García-Porres J, Ortiz-Posadas M. Sigma Level Performance of the Innovated Process in the Imaging Department at a Mexican Health Institute. Journal of Digital Imaging. 2012;26(2):353-360. DOI: https://doi.org/10.1007/s10278-012-9517-z

[58] Pimentel-Aguilar AB, Aguilar-Adaya MK, Sánchez-Castillo EI, Ortiz-Posadas MR: Improving the Drug Dispensing Process at the National Institute of Respiratory Diseases by Applying the Six Sigma Methodology. 33rd Annual International Conference of the IEEE Engineering in Medicine and Biology Society (EMBC). IEEE; Boston 2011. p 1185-1188. DOI: https://doi.org/10.1109/IEMBS.2011.6090278

[59] Becerril-Alquicira A., Ortiz-Posadas MR: Improvement of the Health Technology Management Process of the Public Health Services in Morelos Using the Six Sigma Methodology. 32nd Annual International Conference of the IEEE Engineering in Medicine and Biology Society (EMBC). IEEE; Buenos Aires 2010. p 450-453. DOI: https://doi.org/10.1109/IEMBS.2010.5627370

[60] Thokala P, Devlin N, Marsh K, Baltussen R, Boysen M, Kalo Z et al Multiple Criteria Decision Analysis for Health Care Decision Making-An Introduction: Report 1 of the ISPOR MCDA Emerging Good Practices Task Force. Value in Health. 2016;19(1):1-13. DOI: https://doi.org/10.1016/j.jval.2015.12.003

[61] Rosales-López A, Ortiz-Posadas M. An Indicator to Estimate the Access to Imaging Services in the Costa Rican Public Health System. Journal of Digital Imaging. 2013;27(1):41-48. DOI: https:// doi.org/10.1007/s10278-013-9627-2

[62] Castro H, Tringali M, Cleemput I, Devriese S, Leoni O, Lettieri E. Advancing MCDA and HTA into Coverage Decision-Making. In Marsh K., Goetghebeur M., Thokala P., Baltussen R. (eds) MultiCriteria Decision Analysis to Support Healthcare Decisions. Springer Cham; 2017. p.119-146. DOI: https://doi.org/10.1007/978-3-319-47540-0_8

[63] World Health Organization. Evaluación de tecnologías sanitarias aplicada a los dispositivos médicos. Geneva: WHO; 2012 pp.10. Disponible en: https://apps.who.int/iris/bitstream/han dle/10665/44824/9789243501369_spa.pdf

[64] Aguilar Villanueva LF, Lowi TJ, Allison GT, Linblon CE, Dror Y, Etzioni A, Goodln R, Waldner I, Forester J, Majone G, Meltsner AJ. La hechura de las políticas. México: Miguel Angel Porrúa; 2003 pp. 27

[65] Merino M. Políticas Públicas. Ensayo sobre la intervención del Estado en la solución de problemas públicos. México: CIDE; 2016 pp.17.

[66] Molina G, Roth AN, Muñoz I, Araque J. Políticas públicas en salud: Aproximación a un análisis. Medellín: Facultad Nacional de Salud Pública “Héctor Abad Gómez”, Universidad de Antioquia; 2008 pp.8. 
[67] Lindblom CE. El proceso de elaboración de políticas públicas. Madrid: Ministerio para las Administraciones Públicas; 1991 pp.11.

[68] Augustovski F., Alcaraz A., Caporale J., García Martí S., Pichon Riviere, A. Institutionalizing health technology assessment for priority setting and health policy in Latin America: from regional endeavors to national experiences, Expert Review of Pharmacoeconomics \& Outcomes Research. 2015; 15(1):9-12. DOI: http://dx.doi.org/10.1586/14737167.2014.963560

[69] Pichon-Riviere A, Soto NC, Augustovski FA, García Martí S, Sampietro-Colom L. Evaluación de tecnologías Sanitarias para la toma de decisiones en Latinoamérica: principios de buenas prácticas. Rev Panam Salud Publica. 2017,41: e138. DOI: 10.26633/RPSP.2017.138
[70] Kingdon, J. Agenda, Alternatives and Public Policies. In Shafritz J.M., Layne K.S; Borick C.P. (eds). Classics of Public Policy. New York: Pearson/Longman; 1995.

[71] Demirdjian G. A 10-year hospital-based health technology assessment program in a public hospital in Argentina. International journal of technology assessment in health care. 2015;31(1-2):103-10.

[72] Sixty-seventh World Health Assembly. WHA67.23 - Health Intervention and Technology Assessment in Support of Universal Health Coverage. WHA Resolution. 2014. Disponible en: $h t t p: / /$ apps.who.int/medicinedocs/documents/s21463en/s21463en.pdf. [citado 2019 Jun 24]. 